\title{
Formation Dynamics and Snapshots of Self-injection-locking Dark Solitons
}

\author{
Heming Wang ${ }^{1, \dagger}$, Boqiang Shen ${ }^{1, \dagger}$, Chengying Bao ${ }^{1}$, Warren Jin ${ }^{2}$, Lin Chang ${ }^{2}$, Mark \\ A. Leal ${ }^{2}$, Avi Feshali ${ }^{3}$, Mario Paniccia ${ }^{3}$, John E. Bowers ${ }^{1, *}$, and Kerry Vahala ${ }^{1, *}$ \\ ${ }^{1}$ T. J. Watson Laboratory of Applied Physics, California Institute of Technology, Pasadena, CA 91125, USA. \\ ${ }^{2}$ ECE Department, University of California Santa Barbara, Santa Barbara, CA 93106, USA \\ ${ }^{3}$ Anello Photonics, Santa Clara, CA \\ *jbowers@ucsb.edu,vahala@caltech.edu
}

\begin{abstract}
We propose a model for understanding dark solitons formation dynamics in self-injection-locking laser-resonator systems using dynamical instabilities and domain walls. Snapshots of solitons are captured with dual-comb imaging techniques and validate the model. (c) 2021 The Author(s)
\end{abstract}

Soliton microcombs [1] offer a path towards miniaturization of optical frequency comb technologies. Integration of microcombs with III-V pump lasers [2-4] is an important step towards more fully integrated chip-based soliton microcombs. The self injection locking (SIL) process that accompanies this integration has been shown to create a new operating point that eliminates complex startup and feedback protocols [4]. And recently, SIL has been shown to generate dark solitons in normal-dispersion microresonators [5]. Here we analyze the formation process of such dark solitons in SIL systems. The solitons consist of a pair of domain walls, the spatial counterpart of switching waves previously studied [6]. The domain wall dynamics are governed by energy balance and described by the Maxwell point, which plays a central role in soliton formation and SIL feedback. The model is validated by taking snap-shots of dark soliton shapes via an electro-optic sampling techniques [7].

Fig. 1a shows the SIL laser-resonator system, where a laser cavity is directly connected to a resonator without isolators. Backscattering from the resonator provides feedback to the laser and stabilizes its frequency. In the continuous-wave pumping case, the relationship between the laser detuning $\delta \omega=\omega_{0}-\omega_{\mathrm{P}}\left(\omega_{\mathrm{P}}\right.$ and $\omega_{0}$ are pump frequency and frequency of cavity mode being pumped, respectively) and intracavity power $P_{0}$ can be formulated as [4] $\delta \omega=\left[\tan (\phi / 2)+3 P_{0} /\left(2 P_{\mathrm{th}}\right)\right] \kappa / 2$, where $\kappa$ is the resonator decay rate, $\phi$ is the accumulated phase in the feedback loop and $P_{\text {th }}$ is the parametric oscillation threshold. This peculiar dependency of $\delta \omega$ on $P_{0}$ initially drives the intracavity field to its middle unstable branch of the pumping curve (Fig. 1b). The field randomly evolves to high or low equilibrium points at different positions after a few lifetimes, and to maintain the continuity of the field, domain walls form to connect the high-field and low-field domains (Fig. 1c). Such structures are similar to switching waves [6] that are in the spatial domain and have a topological origin.

Domain walls may shift left or right as a whole in the resonator, and their motion is governed by energy balance. At high- and low-field equilibria, the energy gain derived from pump is balanced by the energy loss rate to the environment. Energy gain dominates for the high-power part of the domain wall, which tends to extend the highfield domain. Meanwhile, energy loss dominates for the low-power part of the domain wall and tends to extend the low-field domain. For the domain wall to remain stationary, these two effects should cancel each other, which imposes the following condition on the pumping power:

$$
\int_{D W}\left(-\kappa|\psi|^{2}+2 \operatorname{Re}\left[f \psi^{*}\right]\right) d \theta=0
$$

where $\psi$ is the intracavity field normalized to energy, $f=\sqrt{\kappa_{\mathrm{ex}} P_{\mathrm{P}}}$ is the pumping term, $\kappa_{e x}$ is the external coupling rate, $P_{\mathrm{P}}$ is the pumping power, and the integration is performed over a single domain wall. For a fixed laser detuning, the unique pump power that stops the domain wall from moving is known as the Maxwell point [8].

In conventionally pumped resonators, where the detuning is fixed, the domain walls keep moving until a pair of oppositely-oriented walls come in contact with each other, at which point the repulsion force between their exponential tails keeps the walls in place. A dark soliton [9] results for pumping above the Maxwell point, while a so-called platicon [10] results for pumping below the Maxwell point. In contrast, for the SIL system, detuning actively tracks the average field of the resonator [4]. If the detuning is away from the Maxwell point, domain walls will move to expand the high- or low-field domain, changing the average intracavity field and therefore pulling the detuning back to the Maxwell point (Fig. 1d). Domain walls may become stationary even without mutual interactions, resulting in a square-wave-like waveform of the dark soliton. And the soliton duty cycle can be adjusted (by tuning the feedback phase or the free-running detuning) to be near $50 \%$ for increased overall comb efficiency, or close to $100 \%$ or $0 \%$ as in conventional dark soliton and platicons, respectively.

To verify the square waveform of the soliton, we generated dark solitons by self-injection locking an InGaAsP DFB laser to a silicon nitride resonator resonator at $1.55 \mathrm{um}$ (free spectral range $10.85 \mathrm{GHz}$ [5]). Snap-shots 

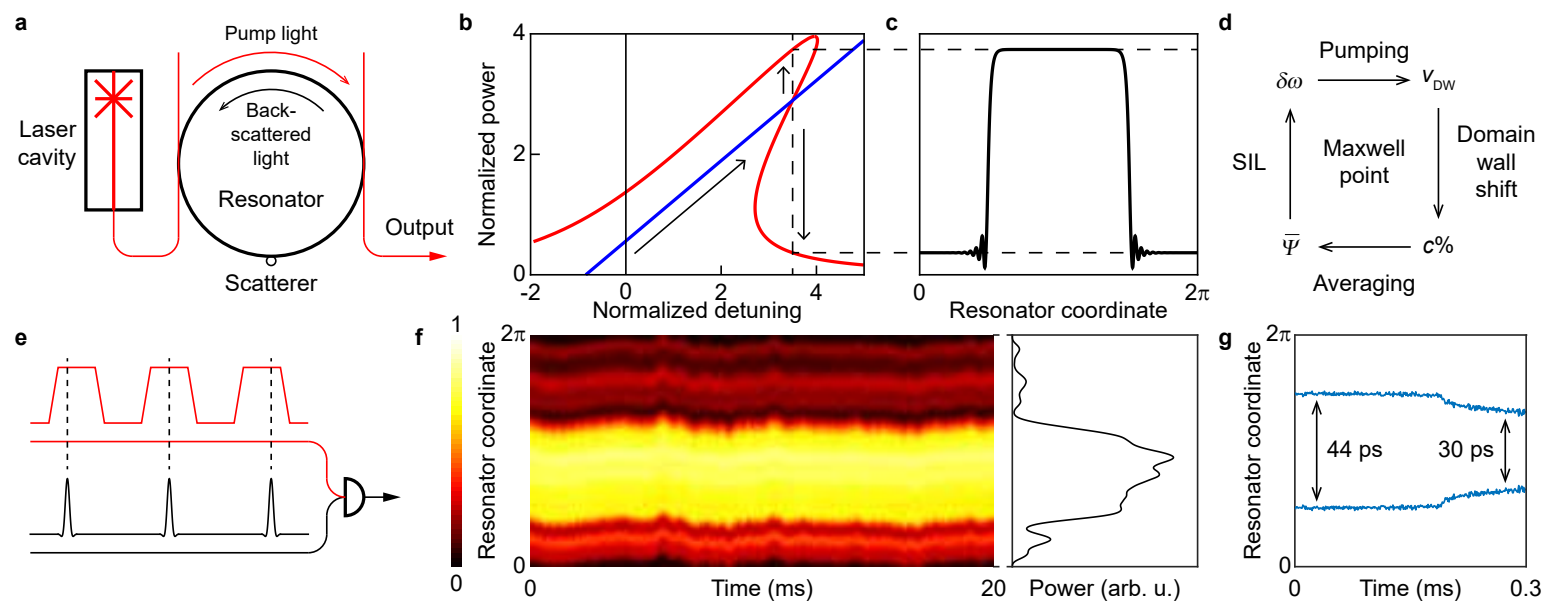

Fig. 1. Dark solitons in microresonators with self injection locking (SIL). (a) Schematic of the SIL setup, consisting of a laser cavity directly coupled to a ring resonator with backscattering. (b) Plot of intracavity power versus detuning for resonator (red) and laser tuning curve with SIL (blue), including the resonator Kerr nonlinearity. Arrows indicate the field evolution process. (c) Simulated dark soliton profiles in the resonator. (d) Diagram for the SIL dark soliton feedback process. $v_{\text {DW }}$ is the domain wall speed, $c \%$ is the duty cycle of the pulse, and $\bar{\psi}$ is the average field in the resonator. (e) Illustration of the soliton imaging process, where the dark soliton is mixed with an EO pulse having a slightly different repetition rate. (f) Measured amplitude (false color) of the field in the resonator when the laser is not scanned. Vertical axis gives the resonator angular coordinate ( 0 to $2 \pi$ ) and horizontal axis is the slow time. Right inset is the power in arbitrary units, averaged over the measurement interval. (g) Position of pulse edges (in resonator angular coordinates) as a function of sweeping time with a scanning laser. Pulse widths are marked at representative scanning times.

of the solitons were obtained by mixing the soliton pulse train at a photodetector with an electro-optically (EO) generated pulse train having a slightly different repetition rate (Fig. 1e). As the pulse trains walk off from each other, the soliton amplitude is sampled at different positions, and the overall soliton shape can be recovered from the detected electric signal [7]. The measured pulse shapes versus evolution time are plotted in Fig. 1f (random drift has been removed). In contrast to the previous bright solitons, dark solitons or platicons, the pulse width can occupy a significant portion of the resonator. Soliton snap-shots were also obtained when sweeping the freerunning laser frequency from blue to red. Within a single scan (outside to inside the resonance), states both with and without dark solitons occur. The pulse width is extracted for a portion of the scan (Fig. 1g). As the detuning $\delta \omega$ increases, the soliton evolves to a state with shorter pulse width and smaller duty cycle, which is consistent with Maxwell-point predictions. The observed jump is believed to result from dispersive-wave effects, which may discretize the positions that the domain walls may occupy.

In summary, we studied and explained the formation of dark solitons in self injection locking systems, and verified predictions by directly measuring the dark soliton pulse shape. The wide range of pulse width and ability to control the width lead to new possibilities for comb applications.

The authors thank Q. Yang, Z. Yuan, and L. Wu for discussions, and DARPA (FA9453-19-C-0029) and AFOSR (FA9550-18-1-0353) for financial support.

\section{References}

1. Kippenberg, T. J., Gaeta, A. L., Lipson, M. \& Gorodetsky, M. L. Science 361, eaan8083 (2018).

2. Stern, B., Ji, X., Okawachi, Y., Gaeta, A. L. \& Lipson, M. Nature 562, 401 (2018).

3. Raja, A. S. et al. Nat. Commun. 10, 680 (2019).

4. Shen, B. et al. Nature 582, 365 (2020).

5. Jin, W. et al. arXiv:2009.07390 (2020).

6. Rozanov, N. N., Semenov, V. E., \& Khodova, G. V. Sov. J. Quantum Electron. 12, 193 (1982).

7. Yi, X., Yang, Q. -F., Yang, K. Y. \& Vahala, K. Nat. Commun. 9, 3565 (2018).

8. Parra-Rivas, P., Gomila, D., Knobloch, E., Coen, S. \& Gelens, L. Opt. Lett. 41, 2402 (2016).

9. Xue, X. et al. Nat. Photon. 9, 594 (2015).

10. Lobanov, V. E., Lihachev, G., Kippenberg, T. J. \& Gorodetsky, M. L. Opt. Express 23, 7713 (2015). 